\title{
Consequences of temporary inhibition of the medial amygdala on social recognition memory performance in mice
}

\author{
Julia Noack, Rita Murau and Mario Engelmann* \\ Center for Behavioral Brain Sciences, Institut für Biochemie und Zellbiologie, Otto-von-Guericke-Universität Magdeburg, \\ Magdeburg, Germany
}

\section{OPEN ACCESS}

Edited by:

Yasushi Kiyokawa,

The University of Tokyo, Japan

Reviewed by:

Sabine M. Hölter,

Helmholtz Zentrum München, German

Research Center for Environmental

Health, Germany

Heather Caldwell,

Kent State University, USA

${ }^{*}$ Correspondence:

Mario Engelmann

AG Neuroendokrinologie and Verhalten, Institut für Biochemie

und Zelllbiologie,

Otto-von-Guericke-Universität Magdeburg, Leipziger Str. 44/Haus 1,

D-39120 Magdeburg, Germany

mario.engelmann@med.ovgu.de

Specialty section:

This article was submitted to Systems Biology,

a section of the journal Frontiers in Neuroscience

Received: 27 January 2015 Accepted: 14 April 2015 Published: 29 April 2015

Citation:

Noack J, Murau R and Engelmann M (2015) Consequences of temporary inhibition of the medial amygdala on

social recognition memory

performance in mice.

Front. Neurosci. 9:152.

doi: 10.3389/fnins.2015.00152
Different lines of investigation suggest that the medial amygdala is causally involved in the processing of information linked to social behavior in rodents. Here we investigated the consequences of temporary inhibition of the medial amygdala by bilateral injections of lidocaine on long-term social recognition memory as tested in the social discrimination task. Lidocaine or control $\mathrm{NaCl}$ solution was infused immediately before learning or before retrieval. Our data show that lidocaine infusion immediately before learning did not affect long-term memory retrieval. However, intra-amygdalar lidocaine infusions immediately before choice interfered with correct memory retrieval. Analysis of the aggressive behavior measured simultaneously during all sessions in the social recognition memory task support the impression that the lidocaine dosage used here was effective as it-at least partially-reduced the aggressive behavior shown by the experimental subjects toward the juveniles. Surprisingly, also infusions of $\mathrm{NaCl}$ solution blocked recognition memory at both injection time points. The results are interpreted in the context of the importance of the medial amygdala for the processing of non-volatile odors as a major contributor to the olfactory signature for social recognition memory.

Keywords: male C57BL/6JOlaHsd mice, social long-term recognition memory, medial amygdala, olfaction, lesion, lidocaine, behavior, learning and memory

\section{Introduction}

Individual recognition in socially living rodents is primarily based on the acquisition and processing of the conspecifics' body odors. They are thought to contain information about age, sex, reproductive state and the health status and, thus, differ individually and they are often referred to as the "olfactory signature." Physico-chemically, body odors are composed of volatile and nonvolatile fractions. Once acquired by the individual, the neuronal processing of such "olfactory signatures" involves distinct brain areas linked to both the main and the accessory olfactory system. One of the most important brain areas involved in the processing of olfactory signals is the medial amygdala (MeA) which has been suggested to play an important role in olfactory social stimulus processing in rodents (Lehman et al., 1980; Ferguson et al., 2001; Broad et al., 2002; Curtis and Wang, 2003; Gobrogge et al., 2007; Gutierrez-Castellanos et al., 2014). Not only the analysis of c-Fos synthesis (Ferguson et al., 2001; Richter et al., 2005; Noack et al., 2010), but also genetic and pharmacological (Ferguson et al., 2001) studies revealed that this brain area may play a key 
role for social recognition in mice. In this context the MeA was shown to act not only as a relay station from the olfactory bulb to deeper brain areas, but also to signal back to the accessory olfactory bulb thereby controlling the impact of the non-volatile fraction of the conspecific's "olfactory signature" on approach-avoidance behavior (Martel and Baum, 2009). However, it is unclear what might be the consequences of temporarily blocking the information processing in the MeA during acquisition/consolidation of olfactory memory vs. its recall. Therefore, we investigated the effects of bilateral temporary suppression of action potential propagation in the $\mathrm{MeA}$ by injecting Lidocaine into the MeA prior to both learning and recall of the individual olfactory information.

Some of the results were previously published in abstract form (Noack et al., 2012).

\section{Materials and Methods}

\section{Experimental Subjects}

Male C57BL/6JOlaHsd mice (originally obtained from HarlanWinkelmann, Bochern, Germany and subsequently bred in the animal facility of the Medical faculty of the Otto-von-GuerickeUniversität Magdeburg), aged 9-16 weeks, were kept in groups of 5 under a constant $12 \mathrm{~h}: 12 \mathrm{~h}$ light-dark cycle (light starting at 6 a.m.) with food and water available ad libitum.

Juvenile C57BL/6JOlaHsd mice of both sexes (age 25-35 days) were used as social stimuli. The suitability of these social stimuli for the behavioral test used was previously proven in intensive studies (Engelmann et al., 2011).

All procedures and manipulations were approved by the local governmental body and according to the European Communities Council Directive recommendations for the care and use of laboratory animals (2010/63/EU).

\section{Social Discrimination Procedure}

Experimental subjects were separated $2 \mathrm{~h}$ before testing. The test procedure consisted of two sessions ( 4 min each) separated by a $24 \mathrm{~h}$ exposition interval (Figure 1A). During the first session (sampling), a conspecific juvenile (J1) was presented to the experimental subject, allowing the adult to acquire the juvenile's "olfactory signature." During the second session (choice) J1 was re-exposed to the adult together with a second, previously not encountered juvenile (J2). A significantly longer investigation duration of J2 vs. J1 during choice was taken as an evidence for an intact long-term social recognition memory (LTsrM) (Engelmann et al., 2011). In addition, sexual (e.g., attempts of mounting) and aggressive behavior [e.g., chasing and biting the juvenile(s)] were separately monitored (see Engelmann et al., 2011 for more details).

\section{Surgery}

Animals were briefly anesthetized with isoflurane (2\%, Baxter, Unterschleißheim, Germany, applied via an anesthesia system (MLW, Leipzig, Germany) by a constant flow of $1.2 \mathrm{l} / \mathrm{min}$ and then injected with a mixture of ketamine and xylazine (i.p., $2 \mathrm{ml}$ Ketavet $^{\circledR}$ (Pfizer Pharmacia, Berlin, Germany), $0.5 \mathrm{ml}$ Rompun ${ }^{\circledR}$ (Bayer Vital, Leverkusen, Germany), $7.5 \mathrm{ml} 0.9 \% \mathrm{NaCl}$ solution).

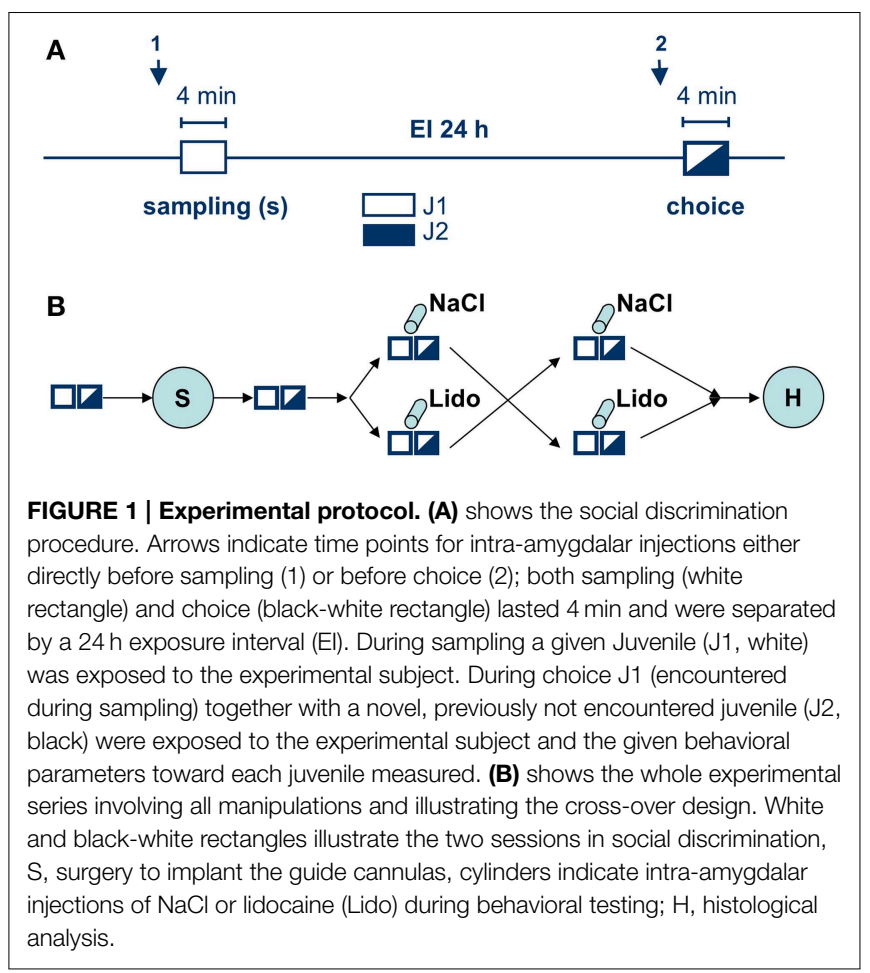

Deeply anesthetized animals were fixed into a stereotaxic frame (TSE Systems, Bad Homburg, Germany). Coordinates for bilateral implantation of the guide cannulas (stainless surgical steel $0.55 \times 0.08 \times 9.00 \mathrm{~mm}$; Injecta, Klingenthal, Germany) were selected according to a stereotaxic mouse brain atlas (Franklin and Paxinos, 1997). The ventral tip of each guide cannula was placed $1 \mathrm{~mm}$ above the target area, the $\mathrm{MeA}$, at the following coordinates starting from Bregma: lateral $\pm 2.5 \mathrm{~mm}$, anterior $0.3 \mathrm{~mm}$, ventral $4.5 \mathrm{~mm}$. The guide cannulas were secured to the skull and to two stainless steel screws $(1.0 \times 2.0 \mathrm{~mm}$, Paul Korth, Lüdenscheid, Germany) inserted into the bone with light curling dental cement (Ivoclar Vivadent, Schaan, Lichtenstein). Stylets were made from insect pins and inserted into the guide cannulas to keep them patent. Animals received an injection of Meloxicam (s.c., $0.05 \mathrm{mg} / \mathrm{kg}$ b. wt., Metacam ${ }^{\circledR}$ Boehringer Ingelheim Vetmedica, Ingelheim, Germany) to temper postoperative pain.

Experimental subjects were allowed to recover from surgery for at least 7 days before the experiment. Pilot studies revealed that at this time point both body weight and social behavior reached pre-surgery levels (Figure 2).

\section{Acute Injections, Substances, and Treatment}

For simultaneous acute bilateral injections animals were removed from the cage, anesthetized with isoflurane (see above) and placed on a table. Stylets were removed and injection cannulas gently inserted into the guide cannulas. Substances were infused via a constant flow of $0.5 \mu \mathrm{l} / \mathrm{min}$ using a microinfusion pump (CMA Microdialysis, Stockholm, Sweden). The proper application was controlled by watching the movement of an airbubble within a scaled tubing connecting the microinfusion pump 


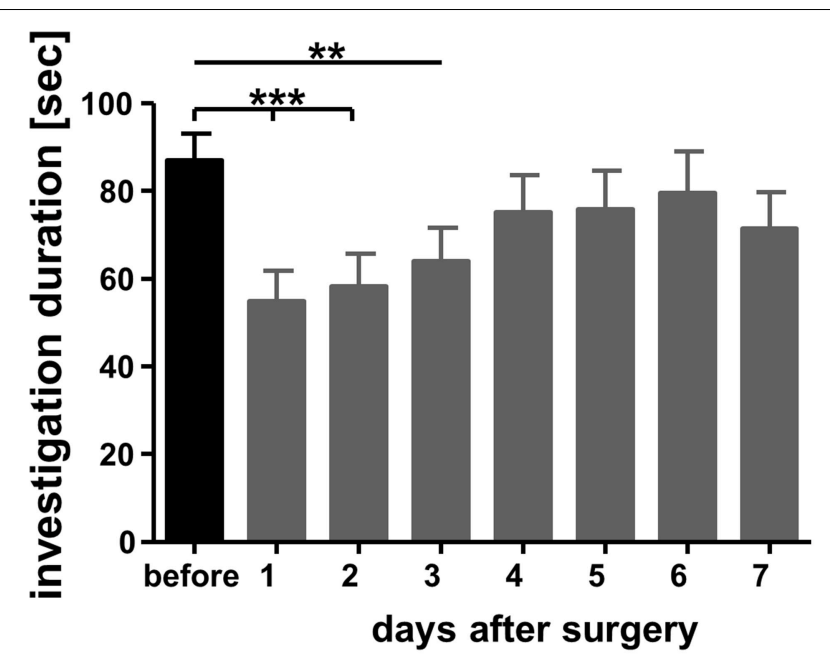

FIGURE 2 | Investigation durations measured a day before bilateral implantation of guide cannulas targeting the medial amygdala and on the subsequent days. Adult male mice were exposed in their home cages for 4 min toward a previously not encountered conspecific juvenile similar to sampling in the social discrimination procedure (for more details see text). A trained observed measured the duration spent by the experimental subjects investigating the juveniles. ${ }^{* *} p<0.01$ and ${ }^{* * *} p<0.01$ compared with the investigation duration measured during "before." Repeated measures ANOVA followed by Dunnett's-test.

with the injection cannula. We infused a volume of $0.5 \mu l$ into each hemisphere. After the infusion injection cannulas were left in place for an additional minute to allow for complete delivery. Subsequently, anesthesia was stopped, the injection cannulas were carefully replaced by the stylets and the animals were returned to their experimental cages. Given the dosage of Lidocaine (Xylocitin-loc ${ }^{\circledR}$, Mibe, Jena, Germany; $20 \mu \mathrm{g} / \mu \mathrm{l}$ diluted in $0.9 \% \mathrm{NaCl}$ solution, Braun, Melsungen, Germany) used we calculated an inhibition of action potential generation and propagation by blocking voltage-gated sodium channels, duration of effect: $<60$ min (Malpeli and Schiller, 1979; Boehnke and Rasmusson, 2001).

\section{Experimental Design}

Before undergoing surgery experimental subjects used in this study were tested for their intact long-term social discrimination abilities. Seven days after surgery the animals were tested for their social discrimination abilities without any further treatment to confirm that the surgery and the implantation of the guide cannulas did not cause effects (e.g., by lesions) that per se interfered with a successful long-term recognition memory.

Recent reports suggest interfering effects of both isoflurane anesthesia (Pearce et al., 2012) and transport of animals between rooms (Moura et al., 2011) on olfactory memory performance. Therefore, we tested the impact of the manipulations linked to the acute intra-amygdalar injections on the behavioral parameters as measured in the present study. Our data revealed that the anesthesia procedure per se did not produce interfering effects for LTsrM if applied according to our experimental protocol (Noack, unpublished observations, Camats Perna, unpublished observations). This is in line with previous reports that the brief isoflurane-anesthesia performed at different time intervals before and after sampling fails to corrupt long-term juvenile recognition abilities in male mice of the mouse strain under study per se (Richter et al., 2005; Wanisch et al., 2008; Engelmann, 2009; Engelmann et al., 2011).

As shown in Figure 1B, infusions of lidocaine or $\mathrm{NaCl}$ were performed in a double-blind, cross-over approach either (1) directly before sampling or (2) directly before choice. The code for the substances was randomly assigned to each experiment separately by a co-worker not involved in the infusion and behavioral testing. The blinding was broken at the end of the histological verification of the placement of the injection side. Recovery time between infusion and the onset of the respective sampling and choice session respectively was $\sim 6 \mathrm{~min}$. The same group of animals ( $n=15$, divided into two subgroups according to the protocol shown in Figure 1B) received the infusion of lidocaine and $\mathrm{NaCl}$ (cross-over) before sampling. Another group of animals ( $n=17$, also divided into two subgroups and treated according to Figure 1B) received the infusions (crossover) before choice. Thus, each animal in the respective group received both lidocaine and $\mathrm{NaCl}$. The interval between the two successive treatments $(\mathrm{NaCl}$ followed by lidocaine or lidocaine followed by $\mathrm{NaCl}$; Figure 1B) was 7 days.

\section{Statistics}

Data are presented as means + SEM. For the analysis of the aggressive/sexual behavior data were submitted in a nonparametric Friedmans ANOVA followed by Wilcoxon corrected for repeated measures. For the analysis of the investigation duration measured during sampling data were analyzed using paired student's $t$-tests. Statistics were performed using GraphPad Prism 4.0 (GraphPad Software Inc., La Jolla, U.S.A.). A $p<0.05$ was considered to indicate statistical significance.

\section{Results}

\section{Aggressive/Sexual Behavior}

We observed that the durations spent in aggressive behavior were-at least under treatment conditions-too low to allow for a reliable analysis (average duration of an investigation bout $<0.5 \mathrm{~s}$ ). Therefore, we analyzed the number of bouts instead since it has been shown to provide a more reliable measure in cases of low durations of individual investigation bouts (Engelmann et al., 2011). Our data show that-compared to implanted, but otherwise untreated conditions - both $\mathrm{NaCl}$ and lidocaine infusions significantly reduced the number of investigation bouts of aggressive/sexual behavior (Friedmans ANOVA: $F s=26.53$, $p<0.01$; Figure 3). Furthermore, under lidocaine treatment aggressive/sexual behavior decreased almost completely reaching statistical significance-compared to $\mathrm{NaCl}$ treatment-if the animals were injected immediately before choice (Friedmans ANOVA: $F s=24.14, p<0.01$; Figure 3).

\section{Social Discrimination}

After injection of $\mathrm{NaCl}$-solution immediately before sampling there was no significant difference in the investigation durations 
of the experimental subjects toward both juveniles $\mathrm{J} 1$ and $\mathrm{J} 2$ during choice (paired $T$-test, $t=1.099, d f=14, p=$ 0.29 ; Figure 4). In contrast, the same animals investigated J2 significantly longer than J1 during choice after receiving the injection of lidocaine directly before sampling (paired $T$-test, $t=2.648, d f=14, p=0.019$ ).

After receiving injections of $\mathrm{NaCl}$ or lidocaine immediately before choice, the experimental subjects showed no significant differences in the duration of investigation behavior toward $\mathrm{J1}$ and $\mathrm{J} 2$ during choice $(\mathrm{NaCl}$ : paired $T$-test, $t=1.270, d f=16$, $p=0.222$; lidocaine: paired $T$-test, $t=1.050, d f=16$, $p=0.309$; Figure 4).

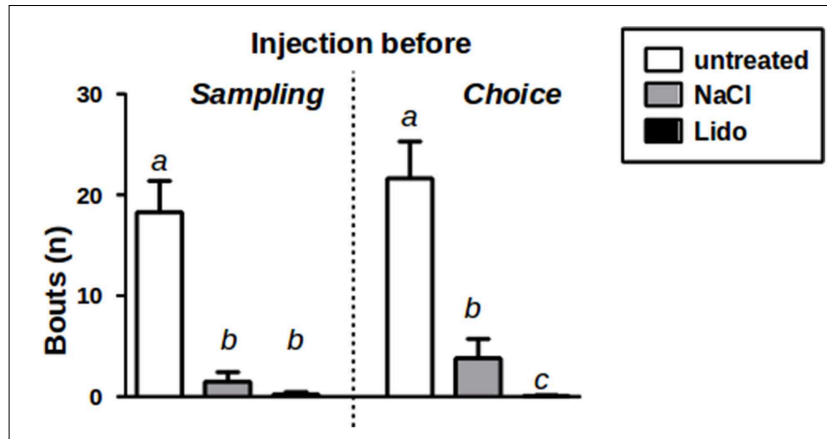

FIGURE 3 | Aggression/Sexual behavior toward J1 during sampling w/o injection (untreated) and after injection of $\mathrm{NaCl}$ or Lidocaine (Lido) directly before sampling (left panel, $\boldsymbol{n}=\mathbf{1 5}$ ). Aggression/Sexual behavior toward $\mathrm{J} 1+\mathrm{J} 2$ during choice $\mathrm{w} / \mathrm{o}$ injection and after injection of $\mathrm{NaCl}$ or Lido directly before choice (right panel, $n=17$ ). $\mathrm{a}=p<0.01 \mathrm{vs}$. $\mathrm{b}$ and $\mathrm{c}$; $\mathrm{c}=$ $p<0.05$ vs. b, Friedman-test followed by Wilcoxon corrected for repeated measures.

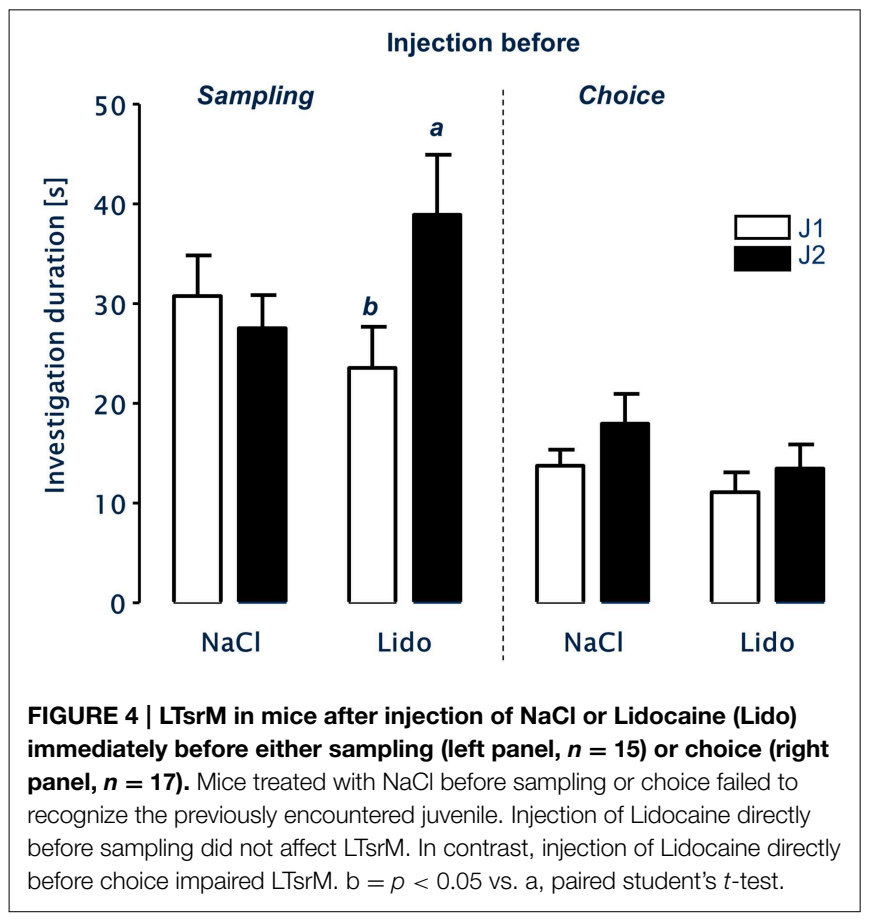

\section{Discussion}

The present study was designed to investigate the impact of temporary inhibition of the medial amygdala on LTsrM. Our data suggest that the combination of isoflurane anesthesia with direct bilateral infusions of different drugs is suitable to investigate the impact for example of temporary lesions of distinct brain areas for LTsrM in mice. More specifically, the protocol used here seems to avoid the interfering effects of isoflurane anesthesia reported by other authors (Pearce et al., 2012).

Previous studies have shown that permanent lesions of the medial amygdala significantly reduced aggressive behavior not only in rats (Vochteloo and Koolhaas, 1987), but also in C57Bl/6 mice (Wang et al., 2013). Aggressive behavior is also seen-albeit in comparably low intensity-during the social discrimination testing where $13-35 \%$ of the total social behavior was considered to be aggressive and of a sexual nature (Engelmann et al., 2011). The almost complete lack of aggressive/sexual behavior toward the stimulus juveniles after administration of lidocaine (see Figure 3) implies that lidocaine administration inhibited local neurosignaling that in turn resulted in a suppression of aggressive/sexual behavior. This observation is important with respect to the effects observed for recognition memory: Lidocaine administration failed to interfere with LTsrM if administered immediately before sampling and blocked the memory performance if administered immediately before choice (Figure 4). Thus, temporarily lesioning the medial amygdala during acquiring the olfactory signature does not affect juvenile recognition.

Interestingly, intra-amygdalar injection of the $\mathrm{NaCl}$-solution alone (originally thought to act as "neutral" control) reduced aggressive behavior (Figure 3) and impaired LTsrM (Figure 4). This implies that the $\mathrm{NaCl}$-solution triggered behavioral effects that tended to be similar to that of lidocaine. Indeed, there is some evidence that infusion of the $\mathrm{NaCl}$-solution also lowers electrophysiological activity in a short-term manner (Malpeli and Schiller, 1979; Tehovnik and Sommer, 1997). Nevertheless, as shown in Figure 3 the behavioral suppressive effect of $\mathrm{NaCl}$ solution treatment was not as pronounced as that of lidocaine. This may have resulted not only in the reduced aggressive activity toward the juveniles (Figure 3), but also in an improper processing of the "olfactory signature" during sampling. This is in line with results of a study investigating among other contextual fear conditioning that revealed that injections of $\mathrm{NaCl}$-solutions into the lateral amygdala mimicked partially effects of lidocaine when compared with sham injections (Calandreau et al., 2005). It may be speculative, but we propose that the resulting modified "olfactory signature" was artificial and could not match with the correctly processed "olfactory signature" obtained during the choice session, resulting in an inability to recognize the originally encountered juvenile, and thus the reported lack of an intact LTsrM.

Lidocaine should completely block electrical activity in the $\mathrm{MeA}$, beyond any abovementioned effect of $\mathrm{NaCl}$, and this is supported by the aggression/sexual behavioral data. We observed that lidocaine injection before sampling did not interfere with LTsrM. However, when injected immediately before choice 
successful recognition of the previously encountered juvenile was blocked (Figure 4). These observations were surprising and led us to the conclusion that information processing within the MeA can be blocked without interfering with the successful acquisition of olfactory information important for LTsrM (electrical block during sampling). According to our results however, the MeA is likely to act as an essential relay station for information during recall after being activated during learning (electrical block only during choice). In addition, an even more detailed interpretation of the different consequences of lidocaine treatment for LTsrM "before sampling" vs. "before choice" may relate to the fact that the $\mathrm{MeA}$ is thought to be primarily involved in the processing of non-volatile olfactory cues (for review see Baum and Bakker, 2013). Previously it was shown that the non-volatile fraction of the "olfactory signature" is not essential for a correct social memory retrieval, if not available during sampling, but it must be available for successful LTsrM during choice when it was accessible during sampling (Noack et al., 2010). Our present results seem to mirror these findings by blocking the information processing of the non-volatile fraction of the olfactory signature in the MeA. Blocking the transfer/processing of information in this brain area by lidocaine, but not $\mathrm{NaCl}$, during learning does not affect LTsrM when both fractions are accessible during retrieval. In contrast, when both fractions of the olfactory signature were processed successfully during sampling (including the non-volatile fraction in the MeA) the blockade (lidocaine) and the modulation $(\mathrm{NaCl})$ of the non-volatile odor processing

\section{References}

Baum, M. J., and Bakker, J. (2013). Roles of sex and gonadal steroids in mammalian pheromonal communication. Front. Neuroendocrinol. 34:268-284. doi: 10.1016/j.yfrne.2013.07.004

Boehnke, S. E., and Rasmusson, D. D. (2001). Time course and effective spread of lidocaine and tetrodotoxin delivered via microdialysis: an electrophysiological study in cerebral cortex. J. Neurosci. Methods 105, 133-141. doi: 10.1016/S01650270(00)00348-4

Broad, K. D., Mimmack, M. L., Keverne, E. B., and Kendrick, K. M. (2002). Increased BDNF and trk-B mRNA expression in cortical and limbic regions following formation of a social recognition memory. Eur. J. Neurosci. 16, 2166-2174. doi: 10.1046/j.1460-9568.2002.02311.x

Calandreau, L., Desmedt, A., Decorte, L., and Jaffard, R. (2005). A different recruitment of the lateral and basolateral amygdala promotes contextual or elemental conditioned association in Pavlovian fear conditioning. Learn. Mem. 12, 383-388. doi: 10.1101/lm.92305

Curtis, J. T., and Wang, Z. (2003). Forebrain c-fos expression under conditions conducive to pair bonding in female prairie voles (Microtus ochrogaster). Physiol. Behav. 80, 95-101. doi: 10.1016/S0031-9384(03)00226-9

Engelmann. (2009). Competition between two memory traces for longterm recognition memory. Neurobiol. Learn. Mem. 91, 58-65. doi: 10.1016/j.nlm.2008.08.009

Engelmann, M., Hädicke, J., and Noack, J. (2011). Testing declarative memory in laboratory rats and mice using the non-conditioned social discrimination procedure. Nat. Protoc. 6, 1152-1162. doi: 10.1038/nprot.2011.353

Ferguson, J. N., Aldag, J. M., Insel, T. R., and Young, L. J. (2001). Oxytocin in the medial amygdala is essential for social recognition in the mouse. J. Neurosci. 21, 8278-8285.

Franklin, K. B. J., and Paxinos, G. (1997). The Mouse Brain in Stereotaxic Coordinates. San Diego, CA: Academic Press. in the MeA interfered with LTsrM. This hypothesis might be an attractive target to further investigate the phenomenon of interference in further studies.

Taken together, the data of the present study suggest that the MeA seems to play an-albeit dispensable-role in the processing of the non-volatile-fraction of the "olfactory signature" for LTsrM. Blocking the generation of action potentials in the MeA during retrieval also blocks LTsrM. Therefore, our data point toward a contribution of the $\mathrm{MeA}$ in the processing of complete "olfactory signatures" for social behavior. These observations illustrate the plasticity of the neuronal substrate for processing stimuli relevant for social recognition memory in order to compensate for the temporary lesion of an otherwise essential brain area.

\section{Author Contributions}

$\mathrm{ME}$ and JN planned the experiments and wrote the manuscript. $\mathrm{JN}$ and RM performed the experiments.

\section{Acknowledgments}

The authors wish to thank PD Dr. Carsten T. Wotjak and Anna Mederer (MPI for Psychiatry Munich) for their supervision with the intracerebral injection procedure in mice and Carole Torsney (Edinburgh) for correcting the English.
Gobrogge, K. L., Liu, Y., Jia, X., and Wang, Z. (2007). Anterior hypothalamic neural activation and neurochemical associations with aggression in pair-bonded male prairie voles. J. Comp. Neurol. 502, 1109-1122. doi: 10.1002/cne. 21364

Gutierrez-Castellanos, N., Pardo-Bellver, C., Martinez-Garcia, F., and Lanuza, E. (2014). The vomeronasal cortex-afferent and efferent projections of the posteromedial cortical nucleus of the amygdala in mice. Eur. J. Neurosci. 39, 141-158. doi: 10.1111/ejn.12393

Lehman, M. N., Winans, S. S., and Powers, J. B. (1980). Medial nucleus of the amygdala mediates chemosensory control of male hamster sexual behavior. Science 210, 557-560. doi: 10.1126/science. 7423209

Malpeli, J. G., and Schiller, P. H. (1979). A method of reversible inactivation of small regions of brain tissue. J. Neurosci. Methods 1, 143-151. doi: 10.1016/0165-0270(79)90011-6

Martel, K. L., and Baum, M. J. (2009). A centrifugal pathway to the mouse accessory olfactory bulb from the medial amygdala conveys gender-specific volatile pheromonal signals. Eur. J. Neurosci. 29, 368-376. doi: 10.1111/j.14609568.2008.06564.x

Moura, P. J., Venkitaramani, D. V., Tashev, R., Lombroso, P. J., and Xavier, G. F. (2011). Transport of animals between rooms: a little-noted aspect of laboratory procedure that may interfere with memory. Behav. Processes 88, 12-19. doi: 10.1016/j.beproc.2011.06.008

Noack, J., Murau, R., and Engelmann, M. (2012). “The medial amygdala in learning and recall of long-term social recognition memory in mice: a temporary lesion study," in FENS Forum Abstr 2012 (Barcelona).

Noack, J., Richter, K., Laube, G., Haghgoo, H. A., Veh, R. W., and Engelmann, M. (2010). Different importance of the volatile and non-volatile fractions of an olfactory signature for individual social recognition in rats versus mice and short-term versus long-term memory. Neurobiol. Learn. Mem. 94, 568-575. doi: 10.1016/j.nlm.2010.09.013 
Pearce, R. A., Duscher, P., Van Dyke, K., Lee, M., Andrei, A. C., and Perouansky, M. (2012). Isoflurane impairs odour discrimination learning in rats: differential effects on short- and long-term memory. Br. J. Anaesth. 108, 630-637. doi: 10.1093/bja/aer451

Richter, K., Wolf, G., and Engelmann, M. (2005). Social recognition memory requires two stages of protein synthesis in mice. Learn. Mem. 12, 407-413. doi: 10.1101/lm.97505

Tehovnik, E. J., and Sommer, M. A. (1997). Effective spread and timecourse of neural inactivation caused by lidocaine injection in monkey cerebral cortex. J. Neurosci. Methods 74, 17-26. doi: 10.1016/S0165-0270(97) 02229-2

Vochteloo, J. D., and Koolhaas, J. M. (1987). Medial amygdala lesions in male rats reduce aggressive behavior: interference with experience. Physiol. Behav. 41, 99-102. doi: 10.1016/0031-9384(87)90137-5

Wang, Y., He, Z., Zhao, C., and Li, L. (2013). Medial amygdala lesions modify aggressive behavior and immediate early gene expression in oxytocin and vasopressin neurons during intermale exposure. Behav. Brain Res. 245, 42-49. doi: 10.1016/j.bbr.2013.02.002

Wanisch, K., Wotjak, C. T., and Engelmann, M. (2008). Long-lasting second stage of recognition memory consolidation in mice. Behav. Brain Res. 186, 191-196. doi: 10.1016/j.bbr.2007.08.008

Conflict of Interest Statement: The authors declare that the research was conducted in the absence of any commercial or financial relationships that could be construed as a potential conflict of interest.

Copyright (C) 2015 Noack, Murau and Engelmann. This is an open-access article distributed under the terms of the Creative Commons Attribution License (CC BY). The use, distribution or reproduction in other forums is permitted, provided the original author(s) or licensor are credited and that the original publication in this journal is cited, in accordance with accepted academic practice. No use, distribution or reproduction is permitted which does not comply with these terms. 